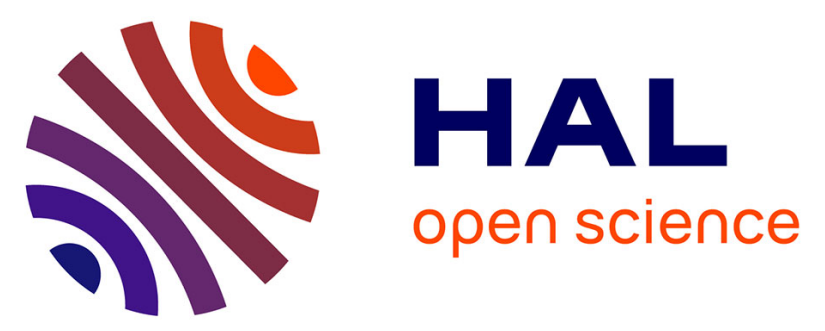

\title{
Number of independent measurements required to obtain reliable mean scattering properties of irregular particles having a small size parameter, using microwave analogy measurements
}

Jean-Baptiste Renard, Jean-Michel Geffrin, Vanesa Tobon Valencia, Hervé

Tortel, François Ménard, Pascal Rannou, Julien Milli, Gwenaël Berthet

\section{To cite this version:}

Jean-Baptiste Renard, Jean-Michel Geffrin, Vanesa Tobon Valencia, Hervé Tortel, François Ménard, et al.. Number of independent measurements required to obtain reliable mean scattering properties of irregular particles having a small size parameter, using microwave analogy measurements. Journal of Quantitative Spectroscopy and Radiative Transfer, 2021, 272, pp.107718. 10.1016/j.jqsrt.2021.107718 . insu-03255038

\section{HAL Id: insu-03255038 \\ https://hal-insu.archives-ouvertes.fr/insu-03255038}

Submitted on 10 Jun 2021

HAL is a multi-disciplinary open access archive for the deposit and dissemination of scientific research documents, whether they are published or not. The documents may come from teaching and research institutions in France or abroad, or from public or private research centers.
L'archive ouverte pluridisciplinaire HAL, est destinée au dépôt et à la diffusion de documents scientifiques de niveau recherche, publiés ou non, émanant des établissements d'enseignement et de recherche français ou étrangers, des laboratoires publics ou privés. 
1 Number of independent measurements required to obtain reliable mean 2 scattering properties of irregular particles having a small size parameter, using

\title{
microwave analogy measurements
}

\author{
Jean-Baptiste Renard ${ }^{1}$, Jean-Michel Geffrin², Vanesa Tobon Valencia ${ }^{2}$, Hervé Tortel ${ }^{2}$, François \\ Ménard $^{3}$, Pascal Rannou ${ }^{4}$, Julien Milli ${ }^{3}$, Gwenaël Berthet ${ }^{1}$ \\ 1. LPC2E, CNRS - Université d'Orléans - CNES, Orléans, France \\ 2. Aix Marseille Université, CNRS, Centrale Marseille, Institut Fresnel, Marseille, France \\ 3. Université Grenoble Alpes, CNRS, IPAG, Grenoble, France \\ 4. GSMA - Université de Reims, Reims, France
}

\begin{abstract}
Laboratory measurements of light scattered by a cloud of randomly oriented levitating particles are often used to interpret remote sensing measurements of dust in space and in Earth's atmosphere. It is necessary to know how many particles or how many different orientations of the same particles must be considered to retrieve the mean scattering function of brightness and polarization. New laboratory measurements were conducted using the microwave analogy method between frequencies of 3 to $18 \mathrm{GHz}$, where an "analog" particle with a small size parameter in a range of 0.5-12 will have a size of several $\mathrm{cm}$. Twelve such "analog" particles from compact shapes to aggregates with small fractal dimensions were fabricated by additive manufacturing (3D printing) and were studied. The number of necessary measurements to reach the mean scattering properties of a particle with an accuracy of about $5 \%$ is obtained for less than 20 different orientations. To reach a $1.5 \%(1-\sigma)$ error in brightness and a $0.5 \%(1-\sigma)$ error in polarization, the number of necessary measurements is in a range of 20 to 70, depending on the shape, fluffiness, deviation from a perfect sphere, and surface irregularities of the particle. These results show that several tens of randomly oriented particles of the same size are sufficient to retrieve the mean light scattering properties. Also, several tens of orientations of the same particles provide mean scattering properties, compared to modelling calculations using the Finite Element Method, for an aggregate composed of identical monomers.
\end{abstract}

\section{Introduction}

Laboratory measurements of light scattered by a cloud of irregular particles are necessary to interpret the brightness and polarization remote sensing measurements of comets, interplanetary dust cloud, and circumstellar material orbiting stars, e.g., protoplanetary and debris disks. Such comparisons are conducted to retrieve bulk physical properties of particles such as composition, size distribution, albedo, and porosity [1-6]. The analyses often assume that the observed particles are randomly oriented inside the cloud, as well as during the reference laboratory measurements. On the opposite, the possible orientation of grains, like in a gas flow or in case of strong magnetic field [7], can modify the scattering properties of an ensemble of particles. Such changes have been shown by [8] during laboratory measurements where the particles were carried and aligned by an airflow, and thus are not considered in this work. 
Several databases of laboratory measurements provide optical properties (phase or scattering functions in intensity and in polarization) of levitating particles having the same size and composition, obtained by different techniques at ground or during microgravity conditions [9-14]. Such experiments, for particles having small to large size parameters $(\pi D / \lambda$, where $D$ is the diameter and $\lambda$ is the wavelength), assume that indeed they are randomly oriented and that enough particles are considered during the measurements, from tens to hundreds or thousands, to retrieve the mean optical properties. As an example, 54 different orientations of the same millimetre-sized particle have been considered by [5] to retrieve the mean scattering properties. Nevertheless, the authors have not quantified in detail how many particles with different orientations are needed to achieve such conditions. Similarly, aerosol counters used in the atmosphere to count and determine the size distribution of the solid particles empirically consider their effective optical properties [16-18]. This approach works well for the submicron particles that are often in large concentrations (more than tens of particles $\mathrm{cm}^{-3}$ ) but some uncertainties in size determination can arise when the concentrations are too low because not enough particles are detected. Finally, experiments and numerical calculations that consider individual irregularly shaped particle need also to know how many different orientations are needed to establish the mean optical properties.

Although theoretical approaches have been proposed [15], experimental approach can also be conducted to determine how many particles randomly oriented, or how many different orientations of the same particle, are indeed necessary to access the mean scattering properties reliably. This approach is difficult to conduct for particles with a small size parameter typically in the range of $0.5-12$, which corresponds to particles between 0.1 to 2.5 $\mu \mathrm{m}$ size in the visible domain. Such particles levitated by an air draught or during microgravity conditions could aggregate, preventing to sustain a cloud of individual particles.

Instead of using real particles, an alternative consists in using the microwave analogy [19-24] and individual particles that can be easily manipulated to control their shape and orientations. The aim will be to measure the properties of a single particle at numerous orientations to figure out its mean properties to mimic an ensemble of specific particles that would be randomly oriented. With that concept, particles of the order of $1 \mu \mathrm{m}$ in the visible domain are of several $\mathrm{cm}$ when studied at a frequency of several $\mathrm{GHz}$, although the size parameter is conserved. Many electromagnetic scattering measurements can be conducted with a single particle by changing only its orientation with respect to the incident radiation beam. Such particles can be built in various materials using a 3-D printer. The permittivity of a given material, or its (complex) refractive index, which is the square root of the (complex) permittivity, can be chosen to reproduce the optical properties of real particles that can be found in space and in Earth's atmosphere.

\section{Conditions of microwave analogy measurements}

The measurements are conducted in the anechoic chamber at the "Centre Commun de Ressources en Microondes" (CCRM, Institut Fresnel, Marseille, France). Features and performances are given in [25]. Briefly, the device operates for this study at 16 frequencies from 3 to $18 \mathrm{GHz}$. The use of 16 frequencies from 3 to $18 \mathrm{GHz}$ allows us to explore a range of size parameters that vary by a factor of six for the same particle, with a refractive index that can be considered almost constant for the chosen materials. The angle between the emitter and receiver antennas is changed from one measurement to another, by steps of $2^{\circ}$, to obtain 
the scattering function in scattering angles from $-130^{\circ}$ to $130^{\circ}$. The sample, mounted at the centre of the device, can be rotated after one session of measurements, to start a new session with another orientation. For the present study, measurements with 36 different orientations uniformly distributed from $-180^{\circ}$ to $+170^{\circ}$ were conducted by a rotation along the vertical axis in steps of $10^{\circ}$. The particles are placed on a polystyrene mast; then the rotation axis is vertical, i.e. parallel to the mast. The particles are placed first according to their greatest length (horizontally), then to their smallest length (vertically), and at about $45^{\circ}$ (slanted) when more measurements are necessary (named inclinations in the following).

The device records the amplitude and the phase of the perpendicular and parallel scattered electric fields. The polarized scattering intensity $I_{\text {perp }}$ and $I_{\text {par }}$ with respect to the scattering plane (also so-called $S$ and $P$ ), are the square of the amplitudes, and are used to retrieve the scattering (or phase) function for the intensity $I$ and for the polarisation $P$ (formulas 1 and 2).

$$
\begin{aligned}
& I=I_{\text {perp }}+I_{\text {par }} \\
& P(\%)=100 \times\left(I_{\text {perp }}-I_{\text {par }}\right) /\left(I_{\text {perp }}+I_{\text {par }}\right)
\end{aligned}
$$

Twelve different samples having a large variety of size, shapes, porosities and fractal dimensions have been studied, which can be representative of the main particle families that can be found in space and Earth's atmosphere. Since particles with different sizes are considered, the present study covers a size parameter range of $0.5-12$. Figure 1 presents the shape of the particles for which some physical parameters are given in table 1 . The definition of the diameter is not easy in case of fluffy particles and particles with low fractal dimension, thus we provide both the gyration diameter and the equivalent diameter calculated from mean projected surface area. The size parameter is calculated from the gyration diameter for the fractal particles (AgC series except AgC181, and SootAcry) and from the equivalent diameter for the compact particles (AgC181, gravels and rough spheres).

For the AgC232, AgC233, AgC185, AgC186 and AgC187, the diameter of the monomer is $5.5 \mathrm{~mm}$. The "SootAcryl" sample is based on a numerically generated analogue of a soot aggregate mimicking necking and overlapping phenomenon [26]. The rough spheres are derived from a perfect sphere meshed with triangles, and then perturbed modifying the distance $d\left(O V_{i}\right)$, with a random perturbation:

$$
d\left(O V_{i}\right)=R+a * r a n d(i)
$$

where $d\left(O V_{i}\right)$ is the distance from the center of the sphere $O$ to the vertex Vi of the triangles, rand is a random number between -1 and 1 , and $R$ is the radius of the original sphere $(16.25$ $\mathrm{mm}$ ). For the "Rough Sphere 233" a= $3.75 \mathrm{~mm}$, and for the "Rough Sphere 228" a = $0.75 \mathrm{~mm}$. The roughness of the sphere and the deviation from sphericity can be estimated by the ratio Di/Dv, where Dv corresponds to the diameter of the smaller compact sphere containing the same amount of material as the particle and Di corresponds of the diameter of the sphere containing the particle.

All the particles are made by additive manufacturing using an acrylate-based resin, except the two gravels that are made of Plexiglas and marble (having similar shapes). The mean refractive index of the particles made of acrylate was measured to be $1.7+0.03 i$ (which can be representative of some carbonaceous particles [27] but also of astrosilicate particles [28]), the refractive index of the Plexiglas is of $1.6+0.01 \mathrm{i}$, and the refractive index of the 
SootAcryl
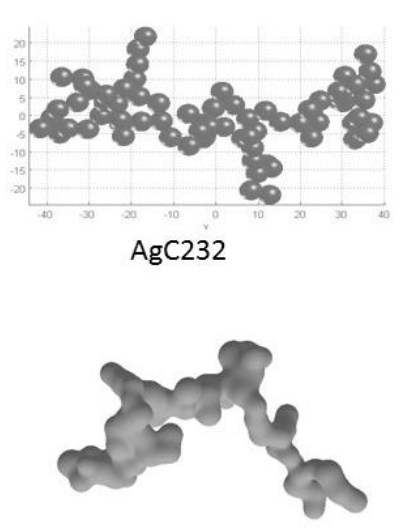

AgC232

marble is assumed to be of $2.9+0.2 \mathrm{i}$ for all the frequencies (which is representative of strongly optically absorbent particles).

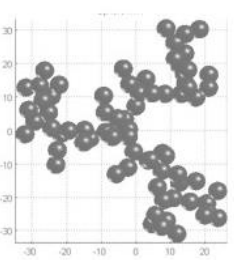

$\mathrm{AgC233}$

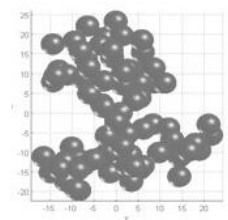

$\mathrm{AgC185}$

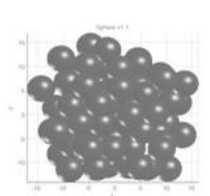

AgC186

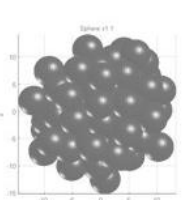

$\mathrm{AgC187}$

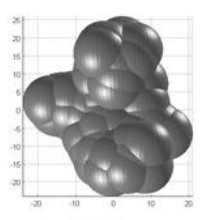

AgC181

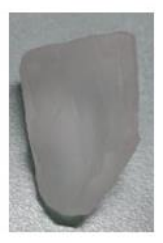

GravelPlexi

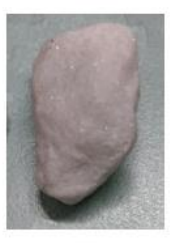

Gravel (marble)

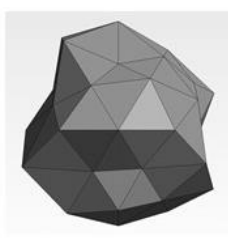

GravelLike

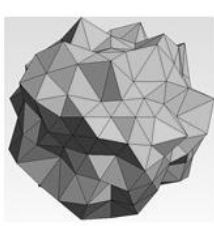

Rough sphere 223

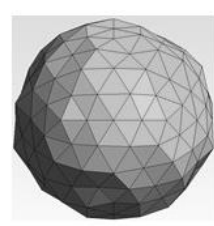

Rough sphere 228

Figure 1: The studied samples (not on the same scale, see table 1 for the size of the samples)

\begin{tabular}{|l|c|c|c|c|c|c|c|}
\hline Sample & $\begin{array}{c}\text { Box containing } \\
\text { the particle } \\
\left(\mathrm{mm}^{3}\right)\end{array}$ & $\begin{array}{c}\text { Gyration } \\
\text { diameter } \\
(\mathrm{mm})\end{array}$ & $\begin{array}{c}\text { Equivalent } \\
\text { diameter } \\
(\mathrm{mm})\end{array}$ & $\begin{array}{c}\text { Fractal } \\
\text { dimension }\end{array}$ & Dv & Di & Di/Dv \\
\hline AgC232 & $76 \times 74 \times 62$ & 64 & 39 & 1.5 & 23.0 & 76 & 3.3 \\
\hline AgC233 & $61 \times 39 \times 67$ & 48 & 37 & 1.7 & 22.9 & 67 & 2.9 \\
\hline AgC185 & $41 \times 43 \times 49$ & 34 & 34 & 2.0 & 23.0 & 49 & 2.1 \\
\hline AgC186 & $34 \times 35 \times 34$ & 23 & 31 & 2.5 & 23.0 & 34 & 1.5 \\
\hline AgC187 & $29 \times 29 \times 29$ & 20 & 27 & 2.8 & 23.0 & 29 & 1.3 \\
\hline AgC181 & $46 \times 28 \times 50$ & - & 40 & - & 35.9 & 50 & 1.4 \\
\hline SootAcryl & $66 \times 48 \times 22$ & 41 & 32 & 1.8 & 22.7 & 68.3 & 3.0 \\
\hline GravelPlexi & $14 \times 20 \times 18$ & - & 17 & - & 14.8 & 24.6 & 1.7 \\
\hline Gravel(marble) & $14 \times 20 \times 18$ & - & 17 & - & 17.0 & 25.3 & 1.5 \\
\hline GravelLike & $32 \times 34 \times 38$ & & 32 & & 30.6 & 38 & 1.2 \\
\hline Rough sphere 223 & $40 \times 40 \times 40$ & - & 36 & - & 31.9 & 40 & 1.3 \\
\hline Rough sphere 228 & $34 \times 34 \times 34$ & - & 34 & - & 32.2 & 34 & 1.05 \\
\hline
\end{tabular}

Table 1: Physical parameters of the samples. The gyration diameters and the fractal idimensions are calculated assuming that the spheres are not interpenetrated; the DV corresponds to diameter of the smaller compact sphere containing the same amount of material as the particle; Di corresponds to the diameter of the sphere containing the particle

\section{Method of measurement analysis}


Individual measurements of the scattering function (intensity and polarization) can exhibit strong oscillations; these oscillations are stronger as the particles are highly irregular. Figures 2 and 3 show examples of such oscillations for the rough sphere 223 and for the AgC185 at a frequency of $12 \mathrm{GHz}$, which corresponds to a size parameter of $\sim 4.5$. The amplitude of the dispersion for intensity at large scattering angles is of the order of 10 for the rough sphere while the amplitude dispersion can reach a factor of 100 for the AgC185. For the polarization, the dispersion can reach at some angles the minimal and maximal possible values $(-100$ and $100 \%)$.
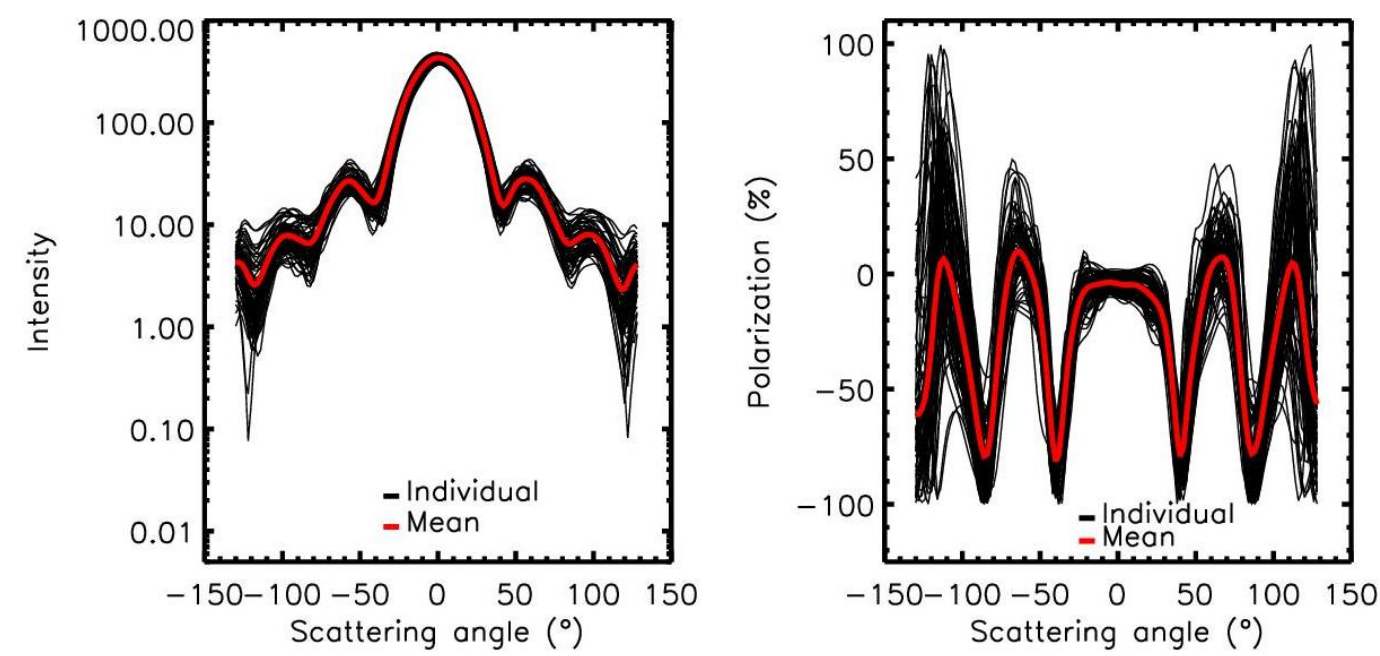

Figure 2: Individual scattering functions for the rough sphere 223 (left: intensity; right: polarization) for the individual measurements at a given orientation and the mean of all the scattering functions
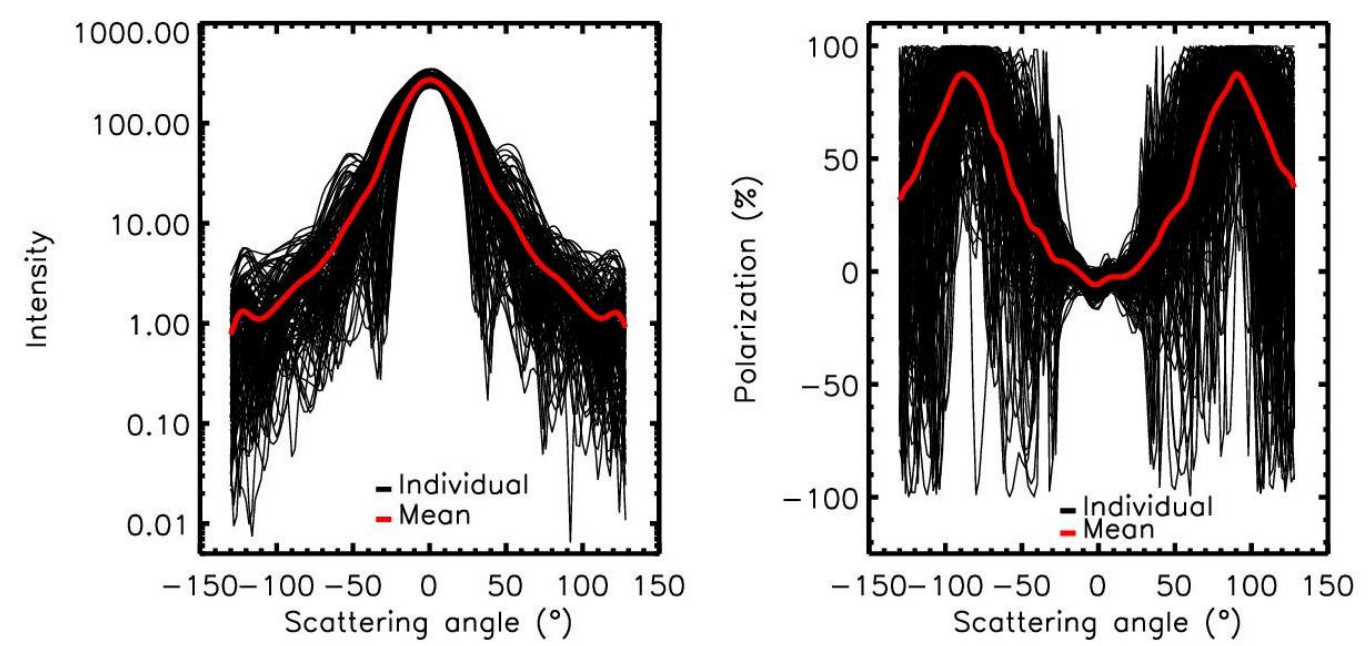

Figure 3: Individual scattering functions for the AgC185 (left: intensity; right: polarization) for the individual measurements at a given orientation and the mean of all the scattering functions 
To determine how many measurements are necessary to retrieve a mean scattering function for intensity and polarization with a given accuracy, tens of individual measurements with different orientations must be considered. The procedure is different for intensity and polarization because intensity is directly retrieved from the sum of polarized measurements while polarization is a relative parameter always retrieved from the ratio between the difference and the sum of polarized intensity measurements and is given in a range from $100 \%$ to $100 \%$.

For the intensity, we calculated the mean scattering functions using a given number of measurements with different orientations. At each scattering angle $\theta$ we calculate the difference $D b(N, \theta)$ between the mean value of the intensity obtained for $\mathrm{N}$ measurements and the mean value obtained for $\mathrm{N}-1$ measurements, divided by the mean value of the intensity for $\mathrm{N}$ measurements; this division act as a normalization to be able to consider simultaneously the results coming for all scattering angles. Then for each value of $\mathrm{N}$, we calculate the standard deviation (hereafter called $\mathrm{SDB}(\mathrm{N})$ ) of the normalized intensity differences obtained for all scattering angles. This standard deviation, which can represent the 1- $\sigma$ uncertainty of the resulting mean intensity values, must decrease while the number of measurements increases. This procedure can be expressed as follows:

$$
\begin{aligned}
& \mathrm{Db}(\mathrm{N}, \theta)=\left(\sum_{1}^{\mathrm{N}} \mathrm{B}(\theta)-\sum_{1}^{\mathrm{N}-1} \mathrm{~B}(\theta)\right) / \sum_{1}^{\mathrm{N}} \mathrm{B}(\theta) \\
& \mathrm{SDB}(\mathrm{N})=\sigma(\operatorname{Db}(\mathrm{N}, \theta))
\end{aligned}
$$

with $\mathrm{N}$ the number of measurements, $\mathrm{B}$ the intensity and $\theta$ the scattering angle.

To ensure a random distribution of the orientation when calculating the $D b(N, \theta)$ parameter, the calculation is conducted for different combinations on the angular orientation and the inclination of the sample; then the results for $\operatorname{SDB}(\mathrm{N})$ are averaged. The choice of the first orientation and the selection of the following orientations (randomly distributed or considering increasing angles) do not statistically change the results for the number of necessary measurements that remains within a $\pm 15 \%$ uncertainty.

As a first step to visualise the sensitivity to the number of measurements, Figure 4 presents the evolution of the standard deviation with the number of measurements (the plot is based on the rotation of the particles around different axes). The frequencies here considered are above the Rayleigh scattering regime (size parameter greater than about 2.5 [29]) and different combinations of orientations and inclinations of the AgC185 sample. In loglog scale, the trend is almost linear, indicating that it could be fitted by a power law that will depend on the studied sample (exponent typically in the -0.8-1.1 range).

Three different domains can be defined; firstly, the standard deviation decreases strongly to reach an accuracy of 0.05 (i.e. $5 \%$ ) for about 15 different orientations, secondly the standard deviation slowly decreases for a number of measurements up to about 70 producing a mean intensity uncertainty of about 0.01 (i.e. 1\%), and thirdly the standard deviation slightly decreases producing a mean intensity uncertainty below 0.01 (i.e. $1 \%$ ). Increasing the number of measurements will reduce again the uncertainties, but such approach does not seem necessary since we can admit that a $1-\sigma$ accuracy of the order of $1 \%$ is sufficient for the interpretation of the intensity measurements. 


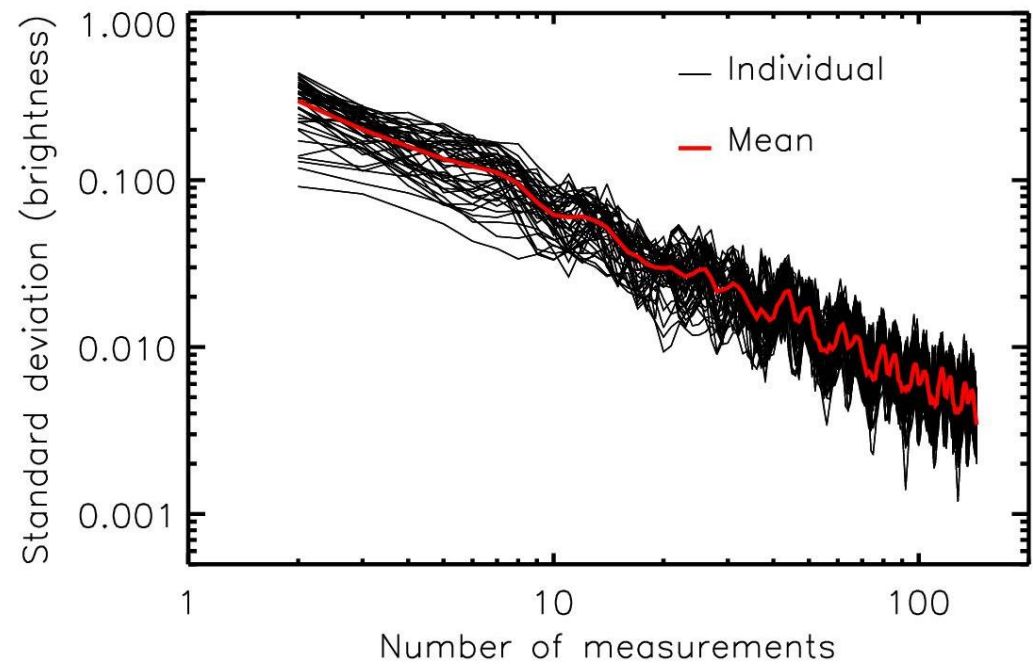

Figure 4: Evolution of the standard deviation with the number of measurements for the intensity of the AgC185 sample; the black lines represent the results for the different frequencies and the different combinations of orientations and inclinations; the red line represents the mean value

For the polarization, the sums of the $\mathrm{N}$ orthogonal and parallel polarized scattering components are first calculated, then the polarization is calculated. The $\operatorname{Dp}(\mathrm{N}, \theta)$ parameter is calculated by the difference between the polarization obtained for $\mathrm{N}$ measurements and the polarization obtained for $\mathrm{N}-1$ measurements. Finally, the SDP(N) parameter corresponds to the standard deviation of $\operatorname{Dp}(\mathrm{N}, \theta)$ for all scattering angles, which corresponds to the polarization uncertainty at $1-\sigma$ in absolute value (\%). This procedure can be expressed as follows:

$$
\begin{aligned}
& \mathrm{P}(\mathrm{N}, \theta)=100 .\left(\sum_{1}^{\mathrm{N}} \mathrm{I}_{\text {perp }}(\theta)-\sum_{1}^{\mathrm{N}} \mathrm{I}_{\mathrm{par}}(\theta)\right) /\left(\sum_{1}^{\mathrm{N}} \mathrm{I}_{\mathrm{per}}(\theta)+\sum_{1}^{\mathrm{N}} \mathrm{I}_{\mathrm{par}}(\theta)\right) \\
& \operatorname{Dp}(\mathrm{N}, \theta)=\mathrm{P}(\mathrm{N}, \theta)-\mathrm{P}(\mathrm{N}-1, \theta) \\
& \operatorname{SDP}(\mathrm{N})=\sigma(\operatorname{Dp}(\mathrm{N}, \theta))
\end{aligned}
$$

Similar trends as those for the intensity are obtained for the polarization measurements, as shown in Figure 5 for the AgC185 sample. A 5\% uncertainty is obtained for about 10 orientations, while about 50 different orientations are necessary to achieve an uncertainty of about $0.5 \%(1-\sigma)$; such last value is of the order of the error bars obtained for some laboratory measurements with real particles $[1,13]$ 


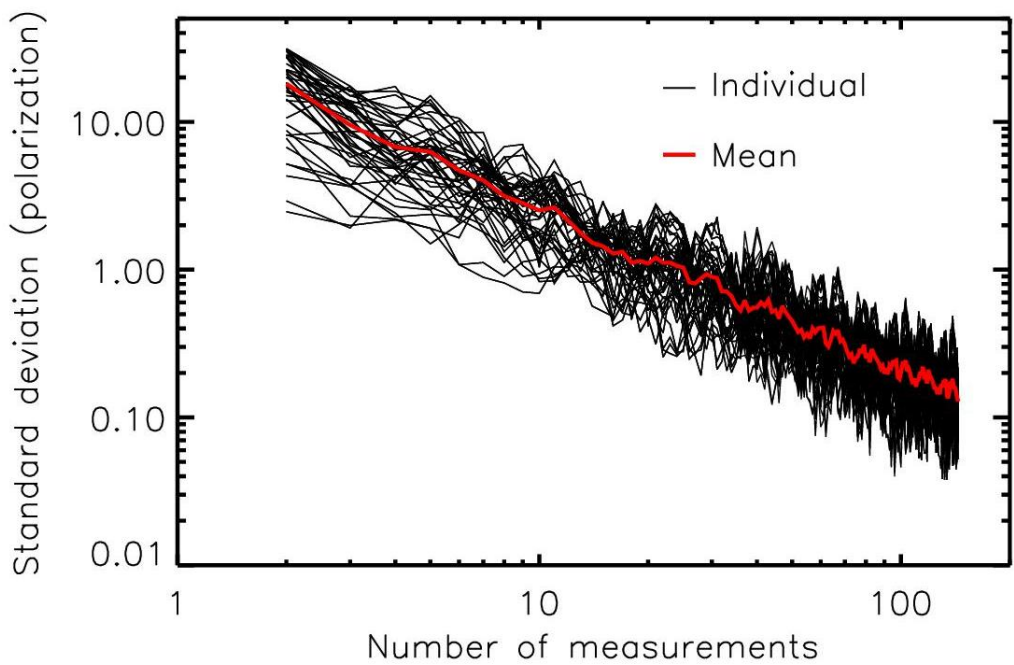

Figure 5: Evolution of the standard deviation for the polarization with the number of measurements of the AgC185 sample; the black lines represent the results for the different frequencies and the different combinations of orientations and inclinations; the red line represents the mean value

\section{Mean scattering functions and evolution with the size parameter}

\subsection{Measurements}

For a perfect sphere, the scattering properties can be easily retrieved using Mie scattering calculations and there is no need for several different orientations of the particle When the roughness and/or the deviation from sphericity of the particle increases, increasing the number of measurements with different orientations of the particle is needed to retrieve the mean properties.

Previous results were calculated for all frequencies above the Rayleigh regime. It is of interest to conduct the studies at each frequency to retrieve the evolution of the standard deviation with the particles' size parameter. For each sample and for all frequencies, the number of necessary measurements where the standard deviation of the intensity drops below $5 \%, 3 \%, 2 \% 1.5 \%$ and $1 \%$ is searched for. Similarly, the number of necessary measurements where the standard deviation of the polarization drops below $5 \%, 3 \%, 2 \%, 1 \%$ and $0.5 \%$ is searched for. The lower values of $1 \%$ for intensity and $0.5 \%$ for polarization are arbitrarily chosen but they can correspond to the typical accuracies often achieved when performing laboratory measurements with levitating particles in the visible spectral domain. All the results for the number of necessary measurements obtained for the different sample inclinations (horizontally, vertically, slated) remains in the $\pm 15 \%$ range for all the standard deviation thresholds presented above.

Figures 6 and 7 present examples of the evolution of the standard deviations with number of measurements for the rough sphere 223 and the $\mathrm{AgC} 185$, having a size parameter of 4.5 . In these examples, the $1 \%$ threshold for intensity is reached for about $35 \pm 5$ and $100 \pm 15$ measurements for the rough sphere 223 and the $\mathrm{AgC185}$, respectively. These values decrease to $30 \pm 5$ and $70 \pm 10$ for the $1.5 \%$ threshold. The $0.5 \%$ threshold for the polarization is reached for $40 \pm 5$ and $70 \pm 10$ for the rough sphere 223 and the $\mathrm{AgC185}$, respectively. These first results indicate that the number of necessary measurements to retrieve the mean optical properties 
(hereafter called necessary measurements) is higher in the case of a non-compact particle compared to a compact one.
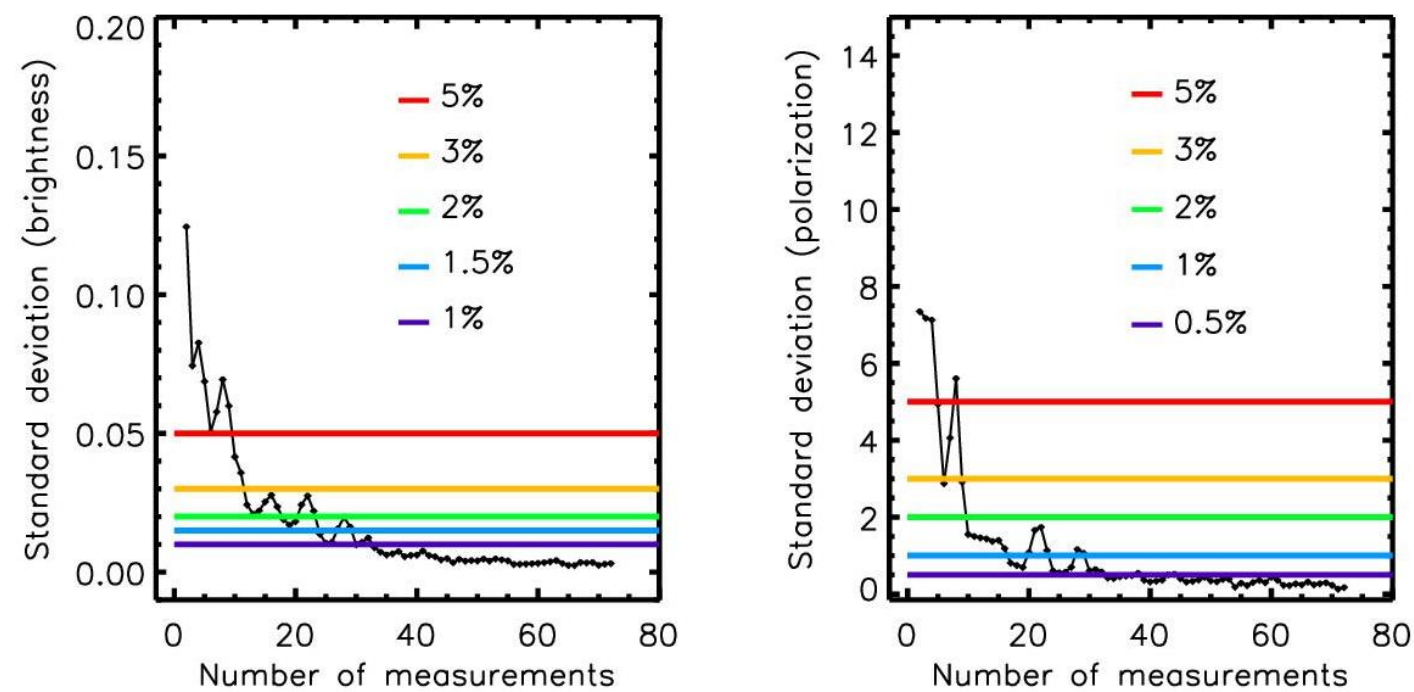

Figure 6: Example of the evolution of the standard deviation for the intensity and polarization with the number of measurements for the rough sphere 223, at a size parameter of 4.5.
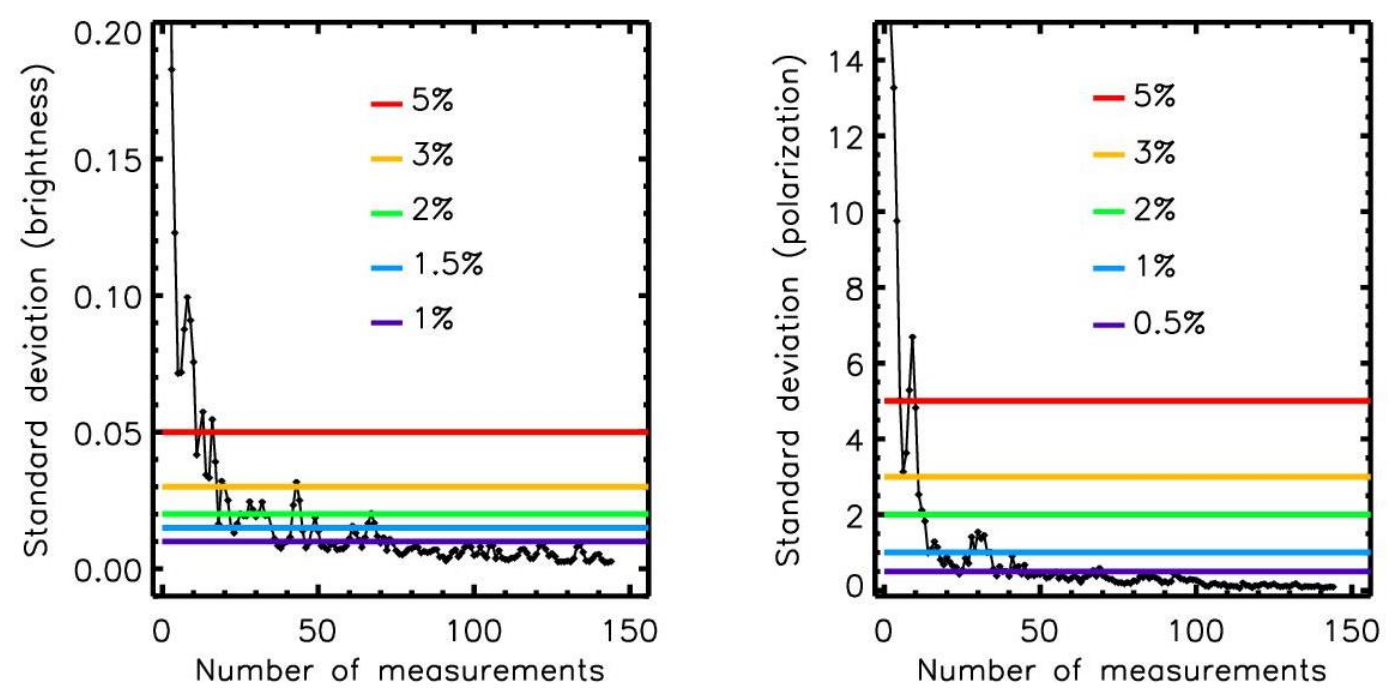

Figure 7: Example of evolution of the standard deviation for the intensity and polarization with the number of measurements for the AgC185, at a size parameter of 4.5.

The evolution of the number of necessary measurements with size parameter for all the samples is presented in Figure 8 for the intensity and in Figure 9 for the polarization, considering the different levels of expected accuracy. Obviously, the number of necessary measurements is lower when the uncertainties is higher, and less than 20 orientations are necessary to retrieve the mean intensity and polarization phase curve with an accuracy of $5 \%$. It also decreases with decreasing size parameter; the decrease is steeper for a size parameter below 3 , corresponding to size particles below the wavelength, and is almost stable for a size parameter greater than 5 . The number converges towards only one necessary measurement when the measurements are performed in the Rayleigh scattering domain. 

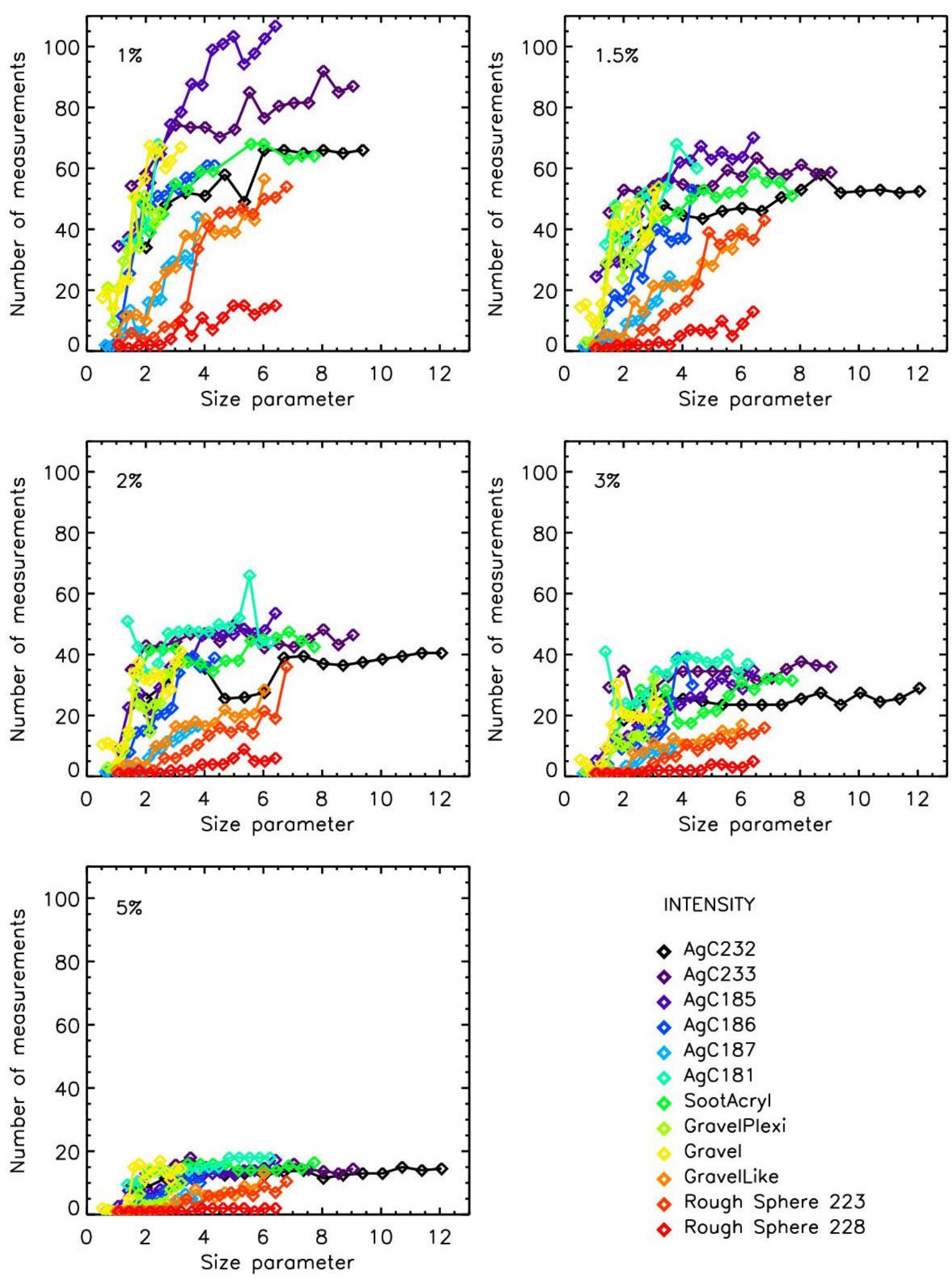

INTENSITY

- $\mathrm{AgC} 232$

- $\mathrm{AgC2} 33$

$\mathrm{AgC} 185$

- $\mathrm{AgC} 186$

- $\mathrm{AgC} 187$

- AgC181

- SootAcryl

GravelPlexi

Gravel

$\diamond$ GravelLike

- Rough Sphere 223

- Rough Sphere 228

Figure 8: Evolution of the number of necessary measurements with size parameters, to retrieve the mean intensity scattering properties, for different thresholds of uncertainties (the error bars of $\pm 15 \%$ for the number of necessary measurements are omitted for clarity). 

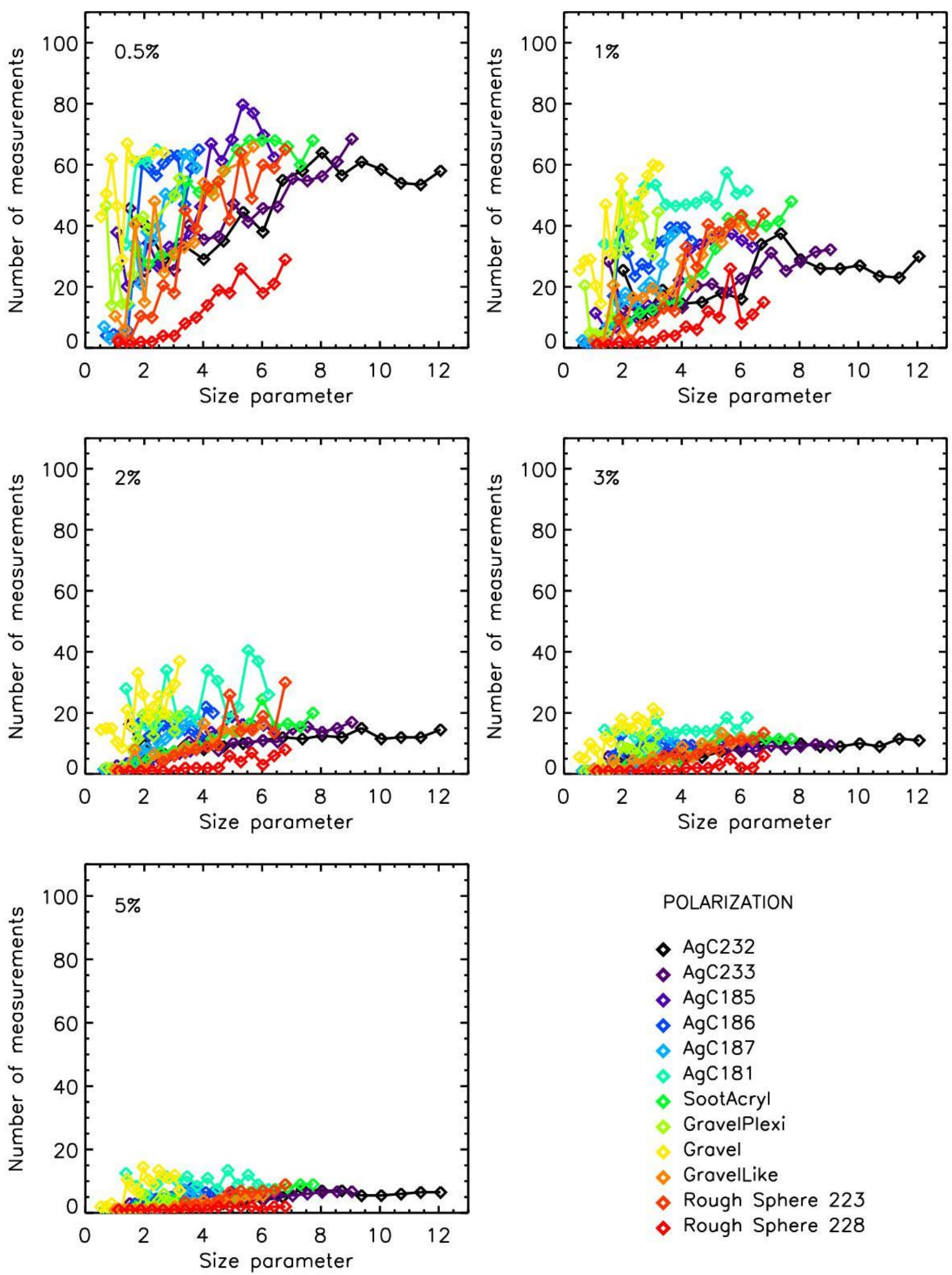

POLARIZATION

- $\mathrm{AgC} 232$

- $\mathrm{AgC} 233$

- $\mathrm{AgC} 185$

- AgC186

$\diamond \mathrm{AgC} 187$

- AgC181

- SootAcryl

- GravelPlexi Gravel

$\diamond$ GravelLike

- Rough Sphere 223

- Rough Sphere 228

Figure 9: Evolution of the number of necessary measurements with size parameters, to retrieve the mean polarization scattering properties, for different thresholds of uncertainties (the error bars of $\pm 15 \%$ for the number of necessary measurements are omitted for clarity). 
The evolution of the necessary number of measurements to reach a given accuracy strongly depends on the nature of the particles. As expected, the lower numbers (around 20 for intensity measurements) are obtained for the near-perfect compact sphere rough sphere 228 , having rugosity in the $[\lambda / 6, \lambda / 33]$ range. Then, the value increases as the surface of the particles become more irregular (rough sphere 223, GravelLike and AgC187) and the shape departs from a sphere. The rough sphere 223 has a particular behaviour, starting from values close to those of the rough sphere 228 for the smallest size parameters and reaching values of more irregular particles for the largest size parameters. This can be understood when considering that roughness depends on the frequencies in the $[\lambda, \lambda / 7]$ range. The other compact particles (Gravel, GravelPlexi, AgC181) and fractal particles (AgC186, SootAcryl, Agc185, Agc232, AgC233) exhibits similar behaviour with the number of necessary measurements in the 40-60 range (mean of 50) for intensity measurements. This categorization for the particles is less obvious when considering the polarization, where only the rough sphere 228 presents a behaviour that stands out from the other sample.

For non-compact particles composed of aggregated monomers, regarding the mean scattering function convergence and the dispersion of the individual scattering function, no direct dependency of the number of necessary measurements with the fractal dimension is detected. Also, the results for the compact Gravel and the GravelPlexi particles are similar, which indicates that for our cases the refractive index has no effect on the number of necessary measurements when the imaginary part of the index indicates significant absorbing properties. For compact particles, the departure from the sphericity reduces the amplitude of Mie oscillations but has little effect on the number of necessary measurements. It seems that the surface roughness, or the size of the irregularities with respect to the wavelength, is the main parameter that drives the necessary number of measurements to retrieve the mean scattering properties. When the surface irregularities or the size of the monomers is below a critical value, probably smaller than about $\lambda / 5$ of the wavelength, they have no effect on the number of necessary measurements that have already reached a constant upper limit. The number of measurements evolves also with the deviation from the sphericity (Di/Dv parameter). When considering particles with size parameter greater than 2.5 , to be above the transition from the Rayleigh scattering regime, the number of measurements reaches its maximum value for Di/DV $>2$ for intensity (accuracy at 1.5\%) and Di/DV > 1.5 for polarization (accuracy at $0.5 \%$ ), as shown in Figure 10.

For the intensity, the $1.5 \%$ accuracy seems a good compromise between the number of necessary measurements and a desired accuracy in this size parameter range. The number of necessary measurements seems to reach an upper limit at about $60 \pm 10$. On the other hand, the fractal aggregates composed of small spherules exhibit small remaining Mie oscillations in the scattering curves even if the number of measurements is large. They produce the small undulations of about $1 \%$ shown in Figure 7 for more than 70 number of measurements, which are difficult to totally remove (more than a hundred of different orientations could then be necessary). For the polarization, an upper limit of $70 \pm 10$ necessary measurements is enough to reach an accuracy of $0.5 \%$ in absolute value. 


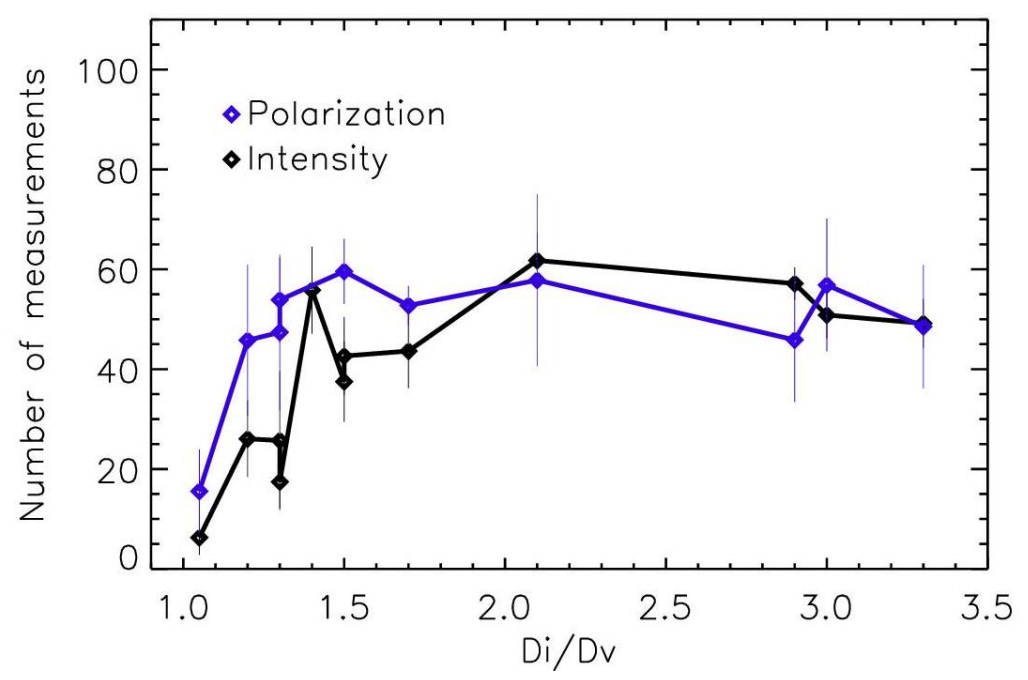

Figure 10: Evolution of the number of measurements as a function of deviation from sphericity (Di/Dv) for particles with size parameter greater than 2.5, for intensity (accuracy at $1.5 \%$ ) and for polarization (accuracy at $0.5 \%$ )

\subsection{Numerical calculations}

Numerical calculations of the scattering properties of the AgC181, AgC185, AgC186, AgC187, AgC232, AgC233 and Gravellike have been conducted using the Finite Element Method presented in [30] and using the same files to describe the geometry of the objects than the one used to print them. The scattering properties are computed considering the similar orientations and inclinations of the sample as during the measurements, by steps of $10^{\circ}$. The same method as the one used for measurements is conducted to determine the number of necessary measurements to retrieve the mean scattering properties.

Figure 11 shows that theoretical calculations and microwave analogy measurements have the same trends and are in correct agreement, considering the uncertainties. Nevertheless, the model for the intensity often requires a larger number of orientations to reach the same error as for the measurements. It seems that the model is more sensitive to the remaining small Mie scattering oscillations than the measurements. The noise existing naturally in the experiments can potentially help to reach more rapidly the random realization. This effect seems less visible in polarization (Figure 12), confirming that intensity and polarization are not sensitive to the same physical parameters of the particles. These results confirm that such modelling calculations can be a good approach to retrieve the mean scattering properties of fluffy and fractal particles when several tens of orientations are considered. 

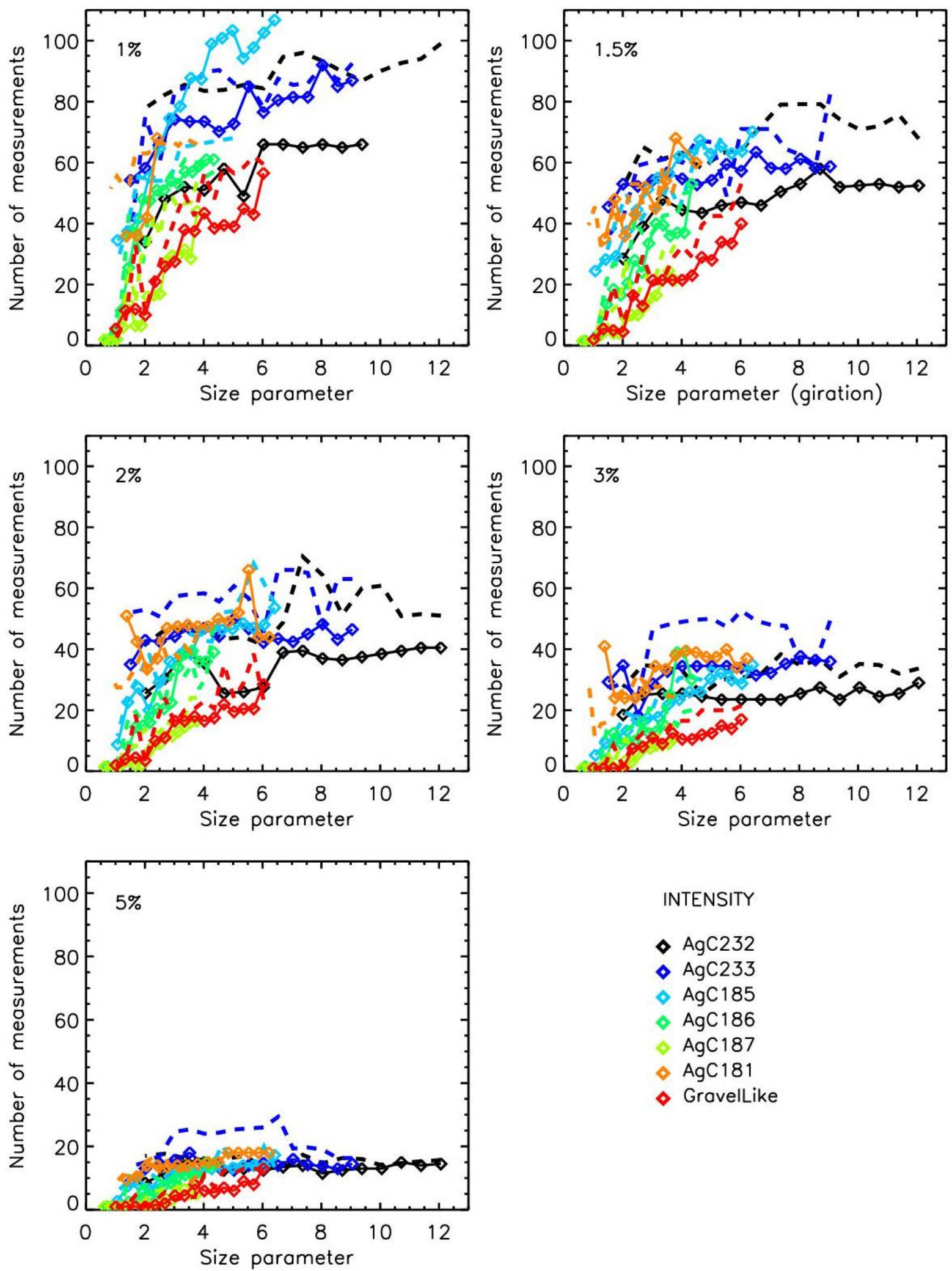

INTENSITY
- $\mathrm{AgC} 232$
- $\mathrm{AgC} 233$
- $\mathrm{AgC} 185$
$\diamond \mathrm{AgC} 186$
- AgC187
$\diamond \mathrm{AgC} 181$
- GravelLike

Figure 11: Evolution of the number of necessary measurements with size parameter, to retrieve the intensity mean optical scattering properties, for different thresholds of uncertainties (the error bars of $\pm 15 \%$ for the number of necessary measurements are omitted for clarity). Full lines: measurements; dotted lines: modelling calculations. 

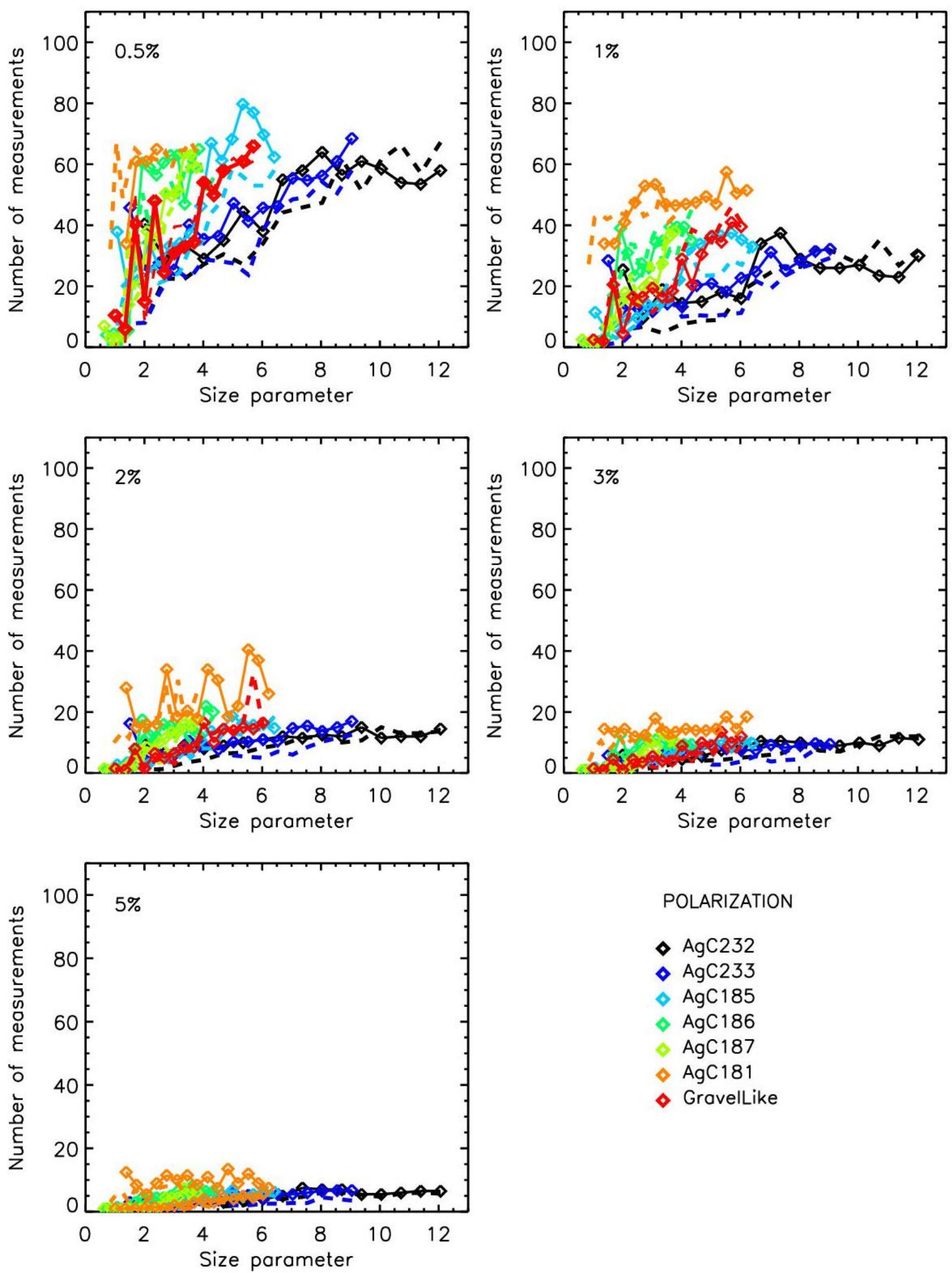

POLARIZATION

$\diamond \mathrm{AgC} 232$

- $\mathrm{AgC} 233$

- AgC185

AgC186

$\mathrm{AgC} 187$

$\mathrm{AgC} 181$

- GravelLike

Figure 12: Evolution of the number of necessary measurements with size parameter, to

retrieve the polarization mean optical scattering properties, for different thresholds of uncertainties (the error bars of $\pm 15 \%$ for the number of necessary measurements are omitted for clarity). Full lines: measurements; dotted lines: modelling calculations. 
The code uses a parallel direct sparse solver (Pardiso); the computation time and memory requirements are strongly related to the object studied and the size of its circumscribing box. Typically, for a run performed on a 28 cores SMP computer, the used memory varies between $7 \mathrm{~GB}$ (AgC187) to $80 \mathrm{~GB}$ (AgC232) while the run time varies between $15 \mathrm{~min}(\mathrm{AgC187})$ to 11 hours (AgC232) for all frequencies. This calculation time for the particles studied here (fractals with less than 75 monomers) is much lower than the tens of hours necessary to retrieve experimentally the mean scattering properties from tens of different orientations. Thus, this numerical approach can be used in complement with real measurements to study the optical response of aggregates with various fractal dimension, number of monomers, shapes, and refractive indexes. On the other hand, experimental measurements could be the only solution to study particles having complex shapes (for examples with different size of monomers or highly variable surface heterogeneities) and to validate new numerical simulations methods or when the number of monomers or size of the objects is higher.

\section{Conclusions}

The microwave analogy has allowed us to estimate how we can define the mean optical scattering properties of irregularly shaped particles observed with different orientations, and how they evolve with the size parameter. For all samples, the number of necessary measurements is between 10 and 20 to reach a mean scattering property with an uncertainty of $5 \%(1-\sigma)$. Then, to reach a $1.5 \%$ error in intensity and $0.5 \%$ in polarisation, the number of necessary measurements strongly depends on the shape, the fluffiness, the deviation from a sphere, and the surface irregularities of the sample with respect to the wavelength (but not directly on their fractal dimension). For a slight rough sphere to a stringlike aggregate of small monomers with a fractal dimension of 1.5, the number of necessary measurements increases from about $20 \pm 3$ to about $70 \pm 10$. The higher value seems to be an upper limit.

Such results corroborate all the previous laboratory measurements of light scattered by clouds of levitating irregular randomly oriented particles where several tens of particles of the same size and having a size parameter below $\sim 10$ are involved. They also show that considering several tens of orientations of the same particle might be sufficient for considering random orientation when performing modelling calculation for an aggregate composed of monomers, thus reducing the calculation time. Finally, new microwave analogy measurements could be conducted to better evaluate the effect of the surface roughness on the mean scattering properties of compact and fluffy particles, in particular for the small size parameters close to the transition to the Rayleigh scattering regime.

Acknowledgments. This work was partly funded by the French PRDA ("Plan Recherche et Développement Amiante") supported by the French Ministry for the Ecological and Inclusive Transition and the Ministry for Regional Cohesion (SU 54-18-054), by the 2019 CNRS 80 Prime programme (project EXPERTS, EXploring Planet formation with lab ExpeRimenTS), by the LOAC-Spatial project funded by the French Space Agency CNES, by the Centre Commun de Ressources en Microondes CCRM, and by the "Groupement de Recherche N³622 SUIE". The authors thanks F. Onifri for the DLA software dedicated to the generation of the aggregates. 


\section{References}

448

1. Hadamcik E, Lasue, J, Levasseur-Regourd AC, Renard, JB. Analogues of interplanetary dust particles to interpret the zodiacal light polarization. Planet Space Sci 2020;183:104527.

2. Levasseur-Regourd AC, Baruteau C, Lasue J, Milli J, Renard JB. Linking studies of tiny meteoroids, zodiacal dust, cometary dust and circumstellar disks. Planet Space Sci 2020;186:104896.

3. Levasseur-Regourd AC, Renard JB, Hadamcik E, Lasue J, Bertini I, Fulle M. Interpretation through experimental simulations of phase functions revealed by Rosetta in 67P dust coma. Astrony Astrophys 3019;630:A20. DOI:10.1051/0004-6361/201834894.

4. Frattin E, Muñoz O, Moreno F, Nava J, Escobar-Cerezo J, Gomez Martin JC, Guirado $D$, Cellino A, Coll P, RaulinF. Experimental phase function and degree of linear polarization of cometary dust analogues. MNRAS 2019 ; 84P :2198-2211. DOI :10.1093/mnras/stz129.

5. Muñoz O, Moreno F, JGómez-Martín JC, Vargas-Martın F, Guirado D, Ramos JL, Bustamante I, Bertini I, Frattin E, Markannen J, Tubiana C, Fulle M, Güttler C, Sierks H, Rotundi A, Della corte V, Ivanovski SL, Zakharov VV, Bockelée-Morvan D, Blum J, Merouane S, Levasseur-Regourd AC, Kolokolova L, Jardiel T, Caballero A. Experimental Phase Function and Degree of Linear Polarization Curves of Millimeter- sized Cosmic Dust Analogs. Astrophysical J Suppl 2020; 247:19. DOI: 10.3847/1538-4365/ab6851.

6. Milli J, Engler N, SchmidH, Olofsson J, Menard F, Kral Q, Boccaletti A, Thébault P, Choquet E, Mouillet D, Lagrange AM, Augereau JC, Pinte C, Chauvin G, Dominik C, Perrot C, Zurlo A, Henning T, Beuzit JL, Avenhaus H, Bazzon A, Moulin T, Llored M, Moeller-Nilsson O, Roelfsema R, Pragt J. Optical polarised phase function of the HR 4796A dust ring. Astron. Astrophys. 2019; 626:A54, DOI: 10.1051/0004-6361/201935363.

7. Andersson BG, Lazarian A, Vaillancourt JE. Interstellar dust grain alignment. Ann. Review Astron. Astrphys. 2015; 53:1, 501-39.

8. Daugeron D, Renard JB, Gaubicher B, Couté B, Hadamcik E, Gensdarmes F, Basso G, Fournier C. Scattering properties of sands, 1. Comparison between different techniques of measurements/ Applied Opt 2006;45:8331-37.

9. Maconi G, Penttilä A, Kassamakov I, Gritsevich M, Helander P, Puranen T, Salmi A, Hæggström E, Muinonen K. Non-destructive controlled single-particle light scattering measurement. J Quant Spectrosc Radiat Transf 2018; 204:159-64.

10. Renard JB, Worms J-C, Lemaire T, Hadamcik E, Huret, N. Light scattering by dust particles in microgravity: polarization and brightness imaging with the new version of the PROGRA2 instrument. Appl Opt 2002; 41(4):609-18. 
11. Renard JB, Hadamcik E, Couté B, Jeannot M, Levasseur-Regourd AC. Wavelength dependence of linear polarization in the visible and near infrared domain for large levitating grains (PROGRA2 instruments). J Quant Spectrosc Radiat Transf 2014; 146:424-30.

12. Hovenier JW, Volten H, Muňoz OK, Van der Zande W, Waters LB. Laboratory studies of scattering matrices for randomly oriented particles: potentials, problems, and perspectives. J. Quant. Spectrosc. Radiat. Transf 2003; 79-80:741-55.

13. Volten $H$, Muňoz $O$, Rol E, de Haan JF, Vassen W, Hovenier JW. Scattering matrices of mineral aerosol particles at 441.6 and 632.8 nm. J.Geophys Res 2001; 106(D15):17375-01.

14. Muňoz O, Moreno F, Guirado D, Dabrowska DD, Volten H, Hovenier JW. The AmsterdamGranada Light Scattering Database. J Quant Spectrosc Radiat Transf 2012; 113(7):564-74.

15. Mishchenko MI, Yurkin MA. On the concept of random orientation in far-field electromagnetic scattering by non-spherical particles. Opt Lett 2017; 42(3):404-97.

16. Grimm H. Eatough DJ. Aerosol measurement: the use of optical light scattering for the determination of particulate size distribution, and particulate mass, including the semi-volatile fraction. J Air Waste Manage Assoc 2009; 59:101-07.

17. Gao RS, Telg H, McLaughlin RJ, Ciciora SJ, Watts LA, Richardson MS, Schwarz JP, Perring $A E$, Thornberry TD, Rollins AW, et al. A light-weight, high-sensitivity particle spectrometer for PM2.5 aerosol measurements. Aeros Sci Technol 2016; 50:88-9.

18. Renard J.B, Dulac F, Berthet G, Lurton T. Vignelles D, égou F, Tonnelier T. Jeannot M, Couté $B$, Akiki R. et al. LOAC, a light aerosols counter for ground-based and balloon measurements of the size distribution and of the main nature of atmospheric particles, Principle of measurements and instrument evaluation. Atmos Meas Technol 2016; 9:172142.

19. Zerull RH, Gustafson BÅS, Schulz K, Thiele-Corbach E. Scattering by aggregates with and without an absorbing mantle: microwave analog experiments. Appl Opt 1993;32: 4088-100. https://doi.org/10.1364/ao.32.004088.

20. Gustafson BÅS, Kolokolova L. A systematic study of light scattering by aggregate particles using the microwave analog technique: Angular and wavelength dependence of intensity and polarization. J Geophys Res Atmos 1999; 104:31711-20. https://doi.org/10.1029/1999JD900327.

21. Sabouroux P, Stout B, Michel Geffrin J, Eyraud C, Ayranci I, Vaillon R, Selçuk N. Amplitude and phase of light scattered by micro-scale aggregates of dielectric spheres: Comparison between theory and microwave analogy experiments. J Quant Spectrosc Radiat Transf 2007; 103:156-67. https://doi.org/10.1016/j.jqsrt.2006.06.001.

22. Merchiers O, Geffrin JM, Vaillon R, Sabouroux P, Lacroix B. Microwave analog to light scattering measurements on a fully characterized complex aggregate. Appl Phys Lett 2009;94:2-4. https://doi.org/10.1063/1.3129196. 
540 23. Merchiers O, Eyraud C, Geffrin J-M, Vaillon R, Stout B, Sabouroux P, et al. Microwave 541 measurements of the full amplitude scattering matrix of a complex aggregate: a database for 542 the assessment of light scattering codes. Opt Express 2010;18:2056. 543 https://doi.org/10.1364/oe.18.002056.

544

545

546

547

548

549

550

551

552

553

554

555

556

557

558

559

560

561

562

563

564

565

566

567

568

569
24. Vaillon R, Geffrin JM. Recent advances in microwave analog to light scattering experiments. J Quant Spectrosc Radiat Transf 2014;146:100-5. https://doi.org/10.1016/j.jqsrt.2014.02.031.

25. Vaillon R, Geffrin JM, Eyraud C, Merchiers O, Sabouroux P, Lacroix B. A new implementation of a microwave analog to light scattering measurement device. J Quant Spectrosc Radiat Transf 2011; 112:1753-60.

26. Yon J, Bescond A, Liu F. On the radiative properties of soot aggregates part 1: Necking and overlapping. J Quant Spectrosc Radiat Transf 2015; 162: 197-206.

27. Bond TC, Bergstrom RW. Light absorption by carbonaceous particles: and investigative review. Aeros Sco Techn 2006; 40: 27-67.

28. Draine BT, Lee HM. Optical properties of interstellar graphite and silicate grains. Astrophys J 1984; 285:89D.

29. van de Hulst H. Light scattering by small particles. John Wiley and Sons eds., New York (USA) 1957.

30. Saleh H, Charon J, Dauchet J, Tortel H, Geffrin JM. Microwave analog experiments on optically soft spheroidal scatterers with weak electromagnetic signature. J Quant Spectrosc. Radiat Transf 2017; 196: 1-9. 